\title{
SmokEng: Towards Fine-grained Classification of Tobacco-related Social Media Text
}

\author{
Kartikey Pant, Venkata Himakar Yanamandra, Alok Debnath and Radhika Mamidi \\ International Institute of Information Technology \\ Hyderabad, Telangana, India \\ \{kartikey.pant, himakar.y, alok.debnath\}@ research.iiit.ac.in \\ radhika.mamidi@iiit.ac.in
}

\begin{abstract}
Contemporary datasets on tobacco consumption focus on one of two topics, either public health mentions and disease surveillance, or sentiment analysis on topical tobacco products and services. However, two primary considerations are not accounted for, the language of the demographic affected and a combination of the topics mentioned above in a finegrained classification mechanism. In this paper, we create a dataset of 3144 tweets, which are selected based on the presence of colloquial slang related to smoking and analyze it based on the semantics of the tweet. Each class is created and annotated based on the content of the tweets such that further hierarchical methods can be easily applied.

Further, we prove the efficacy of standard text classification methods on this dataset, by designing experiments which do both binary as well as multi-class classification. Our experiments tackle the identification of either a specific topic (such as tobacco product promotion), a general mention (cigarettes and related products) or a more fine-grained classification. This methodology paves the way for further analysis, such as understanding sentiment or style, which makes this dataset a vital contribution to both disease surveillance and tobacco use research.
\end{abstract}

\section{Introduction}

As Twitter has grown in popularity to 330 million monthly active users, researchers have increasingly been using it as a source of data for tobacco surveillance (Lienemann et al., 2017). Tobaccorelated advertisements, tweets, awareness posts, and related information is most actively viewed by young adults (aged 18 to 29), who are extensive users of social media and also represent the largest population of smokers in the US and
Canada ${ }^{1}$. Furthermore, it allows us to understand patterns in ethnically diverse and vulnerable audiences (Lienemann et al., 2017). Social media provides an active and useful platform for spreading awareness, especially dialog platforms, which have untapped potential for disease surveillance (Platt et al., 2016). These platforms are useful in stimulating the discussion on societal roles in the domain of public health (Platt et al., 2016). Sharpe et al. (2016) has shown the utility of social media by highlighting that the number of people using social media channels for information about their illnesses before seeking medical care.

Correlation studies have shown that the most probable leading cause of preventable death globally is the consumption of tobacco and tobacco products (Prochaska et al., 2012). The disease most commonly associated with tobacco consumption is lung cancer, with two million cases reported in 2018 alone ${ }^{2}$. While cigarettes are condemned on social media, this has been rivaled by the rising popularity and analysis of the supposed benefits of e-cigarettes (Dai and Hao, 2017). Information pertaining to new flavors and innovations in the industry and surrounding culture have generated sizable traffic on social media as well (Hilton et al., 2016). Studies show that social acceptance is a leading factor to the use and proliferation of e-cigarettes, with some reports claiming as many as 2.39 million high school and 0.63 million middle school students having used an e-cigarette at least once (Malik et al., 2019; Mantey et al., 2019). However, there are strong claims suggesting the use of e-cigarettes as a 'gateway' drug for other illicit substances (Unger et al.,

\footnotetext{
${ }^{1}$ https://www.cdc.gov/tobacco/data statistics/fact_sheets/adult_data/cig_ smoking/

${ }^{2}$ https://www.wcrf.org/dietandcancer/ cancer-trends/lung-cancer-statistics
} 


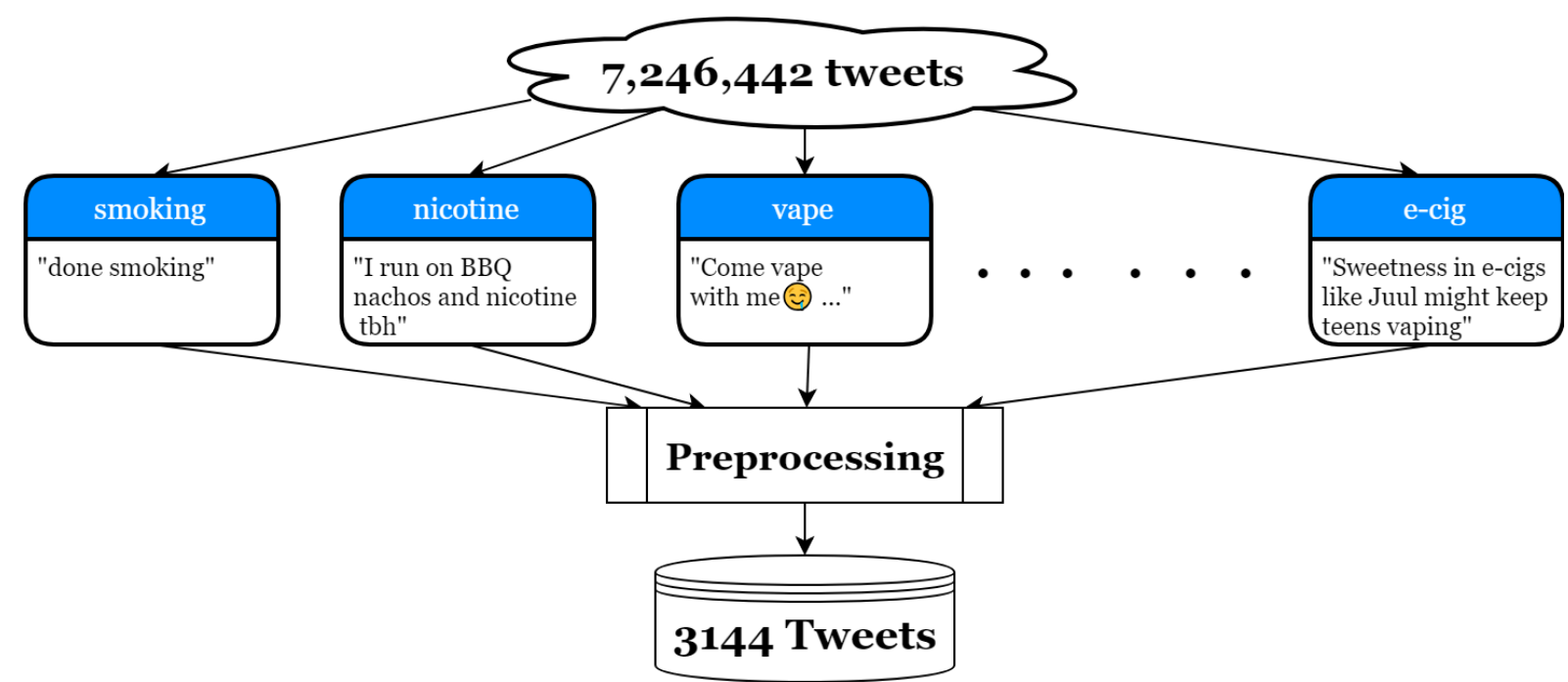

Figure 1: Procedure for Data Collection. We started out with approximately 7 million Tweets which were mined based on 24 slang terms. These were pre-processed to select relevant tweets with decent traction on Twitter. A final cleaned dataset of 3144 tweets is presented.

2016).

In this paper, we aim at classifying tweets relating to cigarettes, e-cigarettes, and other tobaccorelated products into distinct classes. This classification is fine-grained in order to assist in the analysis of the type of tweets which affect the users the most for each product or category. The extensive, manually annotated dataset of 3144 tweets pertains to tobacco use classification into advertisement, general information, personal information, and non-tobacco drug classes. Such classification provides insight into the type of tweet and associated target audience. For example, present cessation programs target users who are ready to quit rather than people who use it regularly, which can be solved using twitter and other online social media (Prochaska et al., 2012). Unlike many previous studies, we also include common slang terms into the classification scheme so as to be able to work with the social media discourse of the target audience.

Finally, we present several text-classification models for the fine-grained classification tasks pertaining to tobacco-related tweets on the released dataset ${ }^{3}$. In doing so, we extend the work in topical Twitter content analysis as well as the study of public health mentions on Twitter.

\section{Related Work}

Myslín et al. (2013) explored content and senti-

\footnotetext{
${ }^{3}$ https://github.com/kartikeypant/ smokeng-tobacco-classification
}

ment analysis of Tobacco-related Twitter posts and performed analysis using machine learning classifiers for the detection of tobacco-relevant posts with a particular focus on emerging products like e-cigarettes and hookah. Their work depends on a triaxial classification along and uses basic statistical classifiers. However, their feature-engineered keyword-based systems do not account for slang associated with tobacco consumption.

Vandewater et al. (2018) performs a classification study based on identifying brand associated with a post using basic text analytics using keywords and image-based classifiers to determine the brands that were most responsible to posting about their brands on social media. Cortese et al. (2018) does a similar analysis on the consumer side, for female smokers on Instagram, targeting the same age group, but based entirely on feature extraction on images, particularly selfies.

More recently, Malik et al. (2019) explored patterns of communication of e-cigarette company Juul use on Twitter. They categorized 1008 randomly selected tweets across four dimensions, namely, user type, sentiment, genre, theme. However, they explore the effects of only Juul, and not other cigarettes or e-cigarettes, further limiting their experiment to only Juul-based analysis and inferences.

In the domain of Disease Surveillance, Aramaki et al. (2011) explored the problem of identifying influenza epidemics using machine-learning based tweet classifiers along with search engine trends 


\begin{tabular}{|c|c|c|}
\hline Name & Label & $\begin{array}{c}\text { Annotation } \\
\text { Class }\end{array}$ \\
\hline Mention of Non-Tobacco Drugs & OD & -1 \\
\hline Unrelated or Ambiguous Mention & $\mathrm{UM}$ & 0 \\
\hline Personal or Anecdotal Mention & $\mathrm{PM}$ & 1 \\
\hline Informative or Advisory Mention & $\mathrm{IM}$ & 2 \\
\hline Advertisements & $\mathrm{AD}$ & 3 \\
\hline
\end{tabular}

Table 1: Label and ID associated with each class.

for medical keywords and medical records for the disease in a local environment. For doing so, they use SVM based classifiers for extracting tweets that mention actual influenza patients. However, since they use only SVM based classifiers, they are limited in their accuracy in classification.

Dai et al. (2017) also focuses on public health surveillance, and uses word embeddings on a topic classifier in order to identify and capture semantic similarities between medical tweets by disease and tweet type for a more robust yet very filtered classification, not accounting for the variety of linguistic features in tweets such as slang, abbreviations and the like in the keyword-based classification mechanism. Jiang et al. (2018) works on a similar problem using machine learning solutions such as an LSTM classifier.

\section{Dataset Creation}

In this section, we explain the development of the dataset that we present along with this paper. We summarize the methods for collecting and filtering through the tweets to arrive at the final dataset and provide some examples of the types of tweets and features we focused on. We also provide the dataset annotation schema and guidelines.

\subsection{Data Collection}

Using the Twitter Application Programming Interface $\left(\mathrm{API}^{4}\right)$, we collected a sample of tweets between 1st October 2018 and 7th October 2018 that represented $1 \%$ of the entire Twitter feed. This $1 \%$ sample consisted of an average 1,035,206 million tweets per day. Out of the 7,246,442 tweets, only tweets written in English and written by users with more than 100 followers have selected for the next step in order to clear spam written by bots.

In order to extract tobacco related tweets from this dataset, we constructed a list of keywords relevant to general tobacco usage, including hookah

\footnotetext{
${ }^{4}$ https://developer.twitter.com/en/products/tweets/sample.html
}

and e-cigarettes. Our initial list consisted of 32 such terms compiled from online slang dictionaries, but we pruned this list to 24 terms. These were smoking, cigarette, e-cig*, cigar, tobacco, hookah, shisha, e-juice, e-liquid, vape, vaping, cheroot, cigarillo, roll-up, ashtray, baccy, rollies, claro, chain-smok*, vaper, ciggie, nicotine, nonsmoker, non-smoking.

By taking the dataset for a full week, we thus avoided potential bias based on the day of the week, which has been observed for alcohol related tweets, which spike in positive sentiment on Fridays and Saturdays (Cavazos-Rehg et al., 2015). For each of the 7 days, all tweets matching any of the listed keywords were included. Tweets matching these tobacco related keywords reflected $0.00043 \%$ of all tweets in the Twitter API 1\% sample. The resulting final dataset thus contained 3144 tweets, with a mean of 449 tweets per day.

\subsection{Data Annotation}

The collected data was then annotated based on the categories mentioned in Table 1. These categories were chosen on the basis of frequency of occurrence, motivated by the general perception of tobacco and non-tobacco drug related tweets. These included advertisements as well anecdotes, information and cautionary tweets. We further noticed that a similar pattern was seen for e-cigarettes and also pertained to some other drugs. While we have explored e-cigarettes in this classification, we have marked the mention of other drugs that were tagged with the same keywords.

A formal definition of each of the categories is given below.

- Unrelated or Ambiguous Mention: This category of tweets contain tweets containing information unrelated to tobacco or any other drug, or pertaining to ambiguity in the intent of the tweet, such as sarcasm. 


\begin{tabular}{|c|l|}
\hline Label & \multicolumn{1}{|c|}{ Examples } \\
\hline $\mathrm{UM}$ & $\begin{array}{l}\text { "What are you smoking bruh ?" } \\
\text { "The smoking gun on Kavanaugh! URL " }\end{array}$ \\
\hline $\mathrm{PM}$ & $\begin{array}{l}\text { "im smoking and doing whats best for me" } \\
\text { "I haven't had a cigarette in \$NUMBER \$ months why do I want one so bad now??" }\end{array}$ \\
\hline $\mathrm{IM}$ & $\begin{array}{l}\text { "Obama puffed. Clinton did cigar feel.Churchill won major wars on whisky." } \\
\text { "The FDA's claim of a teen vaping addiction epidemic doesn't add up. \#ecigarette \#health" }\end{array}$ \\
\hline $\mathrm{AD}$ & $\begin{array}{l}\text { "Which ACID Kuba Kuba are you aiming for? \#De4L \#ExperienceAcid \#cigar \#cigars URL" } \\
\text { "Spookah Lounge: A concept - a year round Halloween-themed hookah lounge" }\end{array}$ \\
\hline $\mathrm{OD}$ & $\begin{array}{l}\text { "Making my money and smoking my weed" } \\
\text { "Mobbin in da Bentley smoking moonrocks." }\end{array}$ \\
\hline
\end{tabular}

Table 2: Examples for each category represented by its label.

- Personal or Anecdotal Mention: Tweets are classified as containing a personal or anecdotal mention if they imply either personal use of tobacco products or e-cigarettes, or provide instances of use of the products by themselves or others.

- Informative or Advisory Mention: This class of tweets consist of a broad range of topics such as:

- mention or discussion on statistics of tobacco and e-cigarette use or consumption

- mention associated health risks or benefits

- portray the use of tobacco products or ecigarettes by a public figure

- emphasize social campaigns for antismoking, smoking cessation and related products such as patches

- Advertisements: All tweets written with the intent of the sale of tobacco products, ecigarettes and associated products or services are marked advertisements. In this classification, intent is considered using the mention of price as an objective measure.

- Mention of Non-Tobacco Drugs: Tweets which mention the use, sale, anecdotes and information about drugs other than ecigarettes or tobacco products are annotated in this category.

\subsection{Inter-annotator Agreement}

Annotation of the dataset to detect the presence of tobacco substance use was carried out by two human annotators having linguistic background and proficiency in English. A sample annotation set consisting of 10 tweets per class was selected randomly from all across the corpus. Both annotators were given the selected sample annotation set. These sample annotation set served as a reference baseline of each category of the text.

In order to validate the quality of annotation, we calculated the Inter-Annotator Agreement (IAA) for the fine-grain classification between the two annotation sets of 3,144 tobacco-related tweets using Cohen's Kappa coefficient (Fleiss and Cohen, 1973). The Kappa score of $\mathbf{0 . 7 9 1}$ indicates that the quality of the annotation and presented schema is productive.

\section{Methodology}

In this section we describe the classifiers designed for this task of fine grained classification. The classifier architecture is based upon a combination of choosing word representations, along with a discriminator that is compatible with that representation. We use the TF-IDF for the suport vector machines and GloVe embeddings (Pennington et al., 2014) with our convolutional neural network architecture and recurrent architectures (LSTM and Bi-LSTM). We also used FastText and BERT embeddings (both base and large) with their native classifiers to note the change in the accuracies.

\subsection{Support Vector Machines (SVM)}

The first learning model used for classification in our experiment was Support Vector Machines (SVM) (Cortes and Vapnik, 1995). We used term frequency-inverse document frequency (TF-IDF) as a feature to classify the annotated tweets in our data set (Salton and Buckley, 1988). TF-IDF cap- 
tures the importance of the given the word in a document, defined in Equation 1.

$$
t f i d f(t, d, D)=f(t, d) \times \log \frac{N}{|\{d \epsilon D: t \epsilon d\}|}
$$

where $f(t, d)$ indicates the number of times term $t$ appears in context, $d$ and $N$ is the total number of documents $|d \epsilon D: t \epsilon d|$ represents the total number of documents where $t$ occurs.

The SVM classifier finds the decision boundary that maximizes the margin by minimizing $\|\mathbf{w}\|$ to find the optimal hyperplane for all the classification tasks:

$$
\begin{gathered}
\min f: \frac{1}{2}\|\mathbf{w}\|^{2} \\
\text { s.t. } \quad y^{(i)}\left(\mathbf{w}^{T} \mathbf{x}^{(i)}+b\right) \geq 1, \quad i=1, \ldots, m
\end{gathered}
$$

where $\mathbf{w}$ is the weight vector, $\mathbf{x}$ is the input vector and $b$ is the bias.

\subsection{Convolutional Neural Networks (CNN)}

In this subsection, we outline the Convolutional Neural Networks (Fukushima, 1988) for classification and also provide the process description for text classification in particular. Convolutional neural networks are multistage trainable neural networks architectures developed for classification tasks (Lecun et al., 1998). Each of these stages consist of the types of layers described below:

- Embedding Layer: The purpose of an embedding layer is to transform the text inputs into a form which can be used by the CNN model. Here, each word of a text document is transformed into a dense vector of fixed size.

- Convolutional Layers: A Convolutional layer consists of multiple kernel matrices that perform the convolution mathematical operation on their input and produce an output matrix of features upon the addition of a bias value.

- Pooling Layers: The purpose of a pooling layer is to perform dimensionality reduction of the input feature vectors. Pooling layers use sub-sampling to the output of the convolutional layer matrices combing neighbouring elements. We have used the commonly used max-pooling function for the pooling.
- Fully-Connected Layer: It is a classic fully connected neural network layer. It is connected to the Pooling layers via a Dropout layer in order to prevent overfitting. Softmax activation function is used for defining the final output of this layer.

The following objective function is commonly used in the task:

$$
E_{w}=\frac{1}{n} \sum_{p=1}^{P} \sum_{j=1}^{N_{L}}\left(o_{j, p}^{L}-y_{j, p}\right)^{2}
$$

where $P$ is the number of patterns, $o_{j, p}^{L}$ is the output of $j^{\text {th }}$ neuron that belongs to $L^{t h}$ layer, $N_{L}$ is the number of neurons in output of $L^{t h}$ layer, $y_{j, p}$ is the desirable target of $j^{\text {th }}$ neuron of pattern $p$ and $y_{i}$ is the output associated with an input vector $x_{i}$ to the $\mathrm{CNN}$.

We use Adam Optimizer (Kingma and Ba, 2014) to minimize the cost function $E_{w}$.

\subsection{Recurrent Neural Architectures}

Recurrent neural networks (RNN) have been employed to produce promising results on a variety of tasks, including language model and speech recognition (Mikolov et al., 2010, 2011; Graves and Schmidhuber, 2005). An RNN predicts the current output conditioned on long-distance features by maintaining a memory based on history information.

An input layer represents features at time $t$. One-hot vectors for words, dense vector features such as word embeddings, or sparse features usually represent an input layer. An input layer has the same dimensionality as feature size. An output layer represents a probability distribution over labels at time $t$ and has the same dimensionality as the size of the labels. Compared to the feedforward network, an RNN contains a connection between the previous hidden state and current hidden state. This connection is made through the recurrent layer, which is designed to store history information. The following equation is used to compute the values in the hidden, and output layers:

$$
\begin{gathered}
\mathbf{h}(t)=f(\mathbf{U x}(t)+\mathbf{W h}(t-1)) . \\
\mathbf{y}(t)=g(\mathbf{V h}(t)),
\end{gathered}
$$

where $U, W$, and $V$ are the connection weights to be computed during training, and $f(z)$ and $g(z)$ 


\begin{tabular}{ccc}
\hline Model/Experiment & Personal Health Mentions & Tobacco-related Mentions \\
\hline$S V M$ & $82.17 \%$ & $83.44 \%$ \\
$C N N$ & $84.08 \%$ & $82.48 \%$ \\
$L S T M$ & $84.39 \%$ & $83.32 \%$ \\
$B i L S T M$ & $83.92 \%$ & $82.97 \%$ \\
$F a s t T e x t$ & $83.76 \%$ & $81.05 \%$ \\
$B E R T_{\text {Base }}$ & $85.19 \%$ & $85.50 \%$ \\
$B E R T_{\text {Large }}$ & $\mathbf{8 7 . 2 6 \%}$ & $\mathbf{8 5 . 6 7 \%}$ \\
\hline
\end{tabular}

Table 3: Binary Classification accuracies for specific topic (Personal Health Mention) or general theme (Tobaccorelated Mentions).

are sigmoid and softmax activation functions as follows.

$$
\begin{aligned}
& f(z)=\frac{1}{1+e^{-z}}, \\
& g\left(z_{m}\right)=\frac{e^{z_{m}}}{\sum_{k} e_{k}^{z}}
\end{aligned}
$$

In this paper, we apply Long Short Term Memory (LSTM) and Bidirectional Long Short Term Memory(Bi-LSTM) to sequence tagging (Hochreiter and Schmidhuber, 1997; Graves and Schmidhuber, 2005; Graves et al., 2013).

LSTM networks use purpose-built memory cells to update the hidden layer values. As a result, they may be better at finding and exploiting long-range dependencies in the data than a standard RNN. The following equation implements the LSTM model:

$$
i_{t}=\sigma\left(W_{x i} x_{t}+W_{h i} h_{t-1}+W_{c i} c_{t-1}+b_{i}\right)
$$

$$
f_{t}=\sigma\left(W_{x f} x_{t}+W_{h f} h_{t-1}+W_{c f} c_{t-1}+b_{f}\right)
$$

$$
o_{t}=\sigma\left(W_{x o} x_{t}+W_{h o} h_{t-1}+W_{c o} c_{t}+b_{o}\right)
$$

$$
h_{t}=o_{t} \tanh \left(c_{t}\right)
$$

In sequence tagging task, we have access to both past and future input features for a given time. Thus, we can utilize a bidirectional LSTM network (Bi-LSTM) as proposed in (Graves et al., 2013).

\subsection{FastText}

FastText classifier has proven to be efficient for text classification (Joulin et al., 2016). It is often at par with deep learning classifiers in terms of accuracy, and much faster for training and evaluation. FastText uses bag of words and bag of n-grams as features for text classification. Bag of n-grams feature captures partial information about the local word order. FastText allows updating word vectors through back-propagation during training allowing the model to fine-tune word representations according to the task at hand (Bojanowski et al., 2016). The model is trained using stochastic gradient descent and a linearly decaying learning rate.

\subsection{BERT}

While previous studies on word representations focused on learning context-independent representations, recent works have focused on learning contextualized word representations. One of the more recent contextualized word representation is BERT (Devlin et al., 2019).

BERT is a contextualized word representation model, pre-trained using bidirectional transformers(Vaswani et al., 2017). It uses a masked language model that predicts randomly masked in a sequence. It uses the task of next sentence prediction for learning the embeddings with a broader context. It outperforms many existing techniques on most NLP tasks with minimal task-specific architectural changes. It is pretrained on 3.3B words from various sources including BooksCorpus and the English Wikipedia.

Based on the transformer architecture used, BERT is classified into two types: $B E R T_{B a s e}$ and $B E R T_{\text {Large. }} B E R T_{\text {Base }}$ uses a 12-layered transformer with $110 \mathrm{M}$ parameters. $B E R T_{\text {Large }}$ uses a 24-layered transformer with $340 \mathrm{M}$ parameters. We use the cased variant of both models. 


\begin{tabular}{llll}
\hline Methods & Accuracy & F1 Score & Recall \\
\hline$S V M$ & $65.45 \%$ & 0.678 & 0.657 \\
$C N N$ & $66.72 \%$ & 0.668 & 0.599 \\
LSTM & $64.97 \%$ & 0.641 & 0.583 \\
BiLSTM & $65.29 \%$ & 0.643 & 0.597 \\
FastText & $69.43 \%$ & 0.696 & 0.669 \\
BERT $_{\text {Base }}$ & $70.86 \%$ & 0.708 & 0.709 \\
BERT $_{\text {Large }}$ & $\mathbf{7 1 . 3 4 \%}$ & $\mathbf{0 . 7 1 4}$ & $\mathbf{0 . 7 1 3}$ \\
\hline
\end{tabular}

Table 4: Evaluation scores for the Fine-grained classification experiment.

\section{Experiments}

In this section, we describe three experiments on the dataset created in the section above. The experiments are designed to show how well existing models perform on the naive binary classification based on this dataset as well as the fine-grained five-class classification system. The first experiment is based on detecting just personal or anecdotal mentions. The second is based on identifying whether a tweet is about tobacco or not. The last experiment is a full fine-grained classification experiment.

The following experiments were conducted keeping an 80-20 split between training and test data, with 2517 tweets in the training dataset and 629 tweets in the test dataset. All tweets were shuffled randomly before the train-test split.

$B E R T_{\text {Large }}$ was observed to perform the best in all three experiments, followed closely by $B E R T_{\text {Base }}$ in all the experiments that were conducted.

\subsection{Experiment 1: Detecting Personal Mentions of Tobacco Use}

The first experiment in the study was to detect tweets containing personal mentions of tobacco use. Tweets containing personal mentions of tobacco use are the ones marking implicit or explicit use of a tobacco substance by the poster. The objective of this experiment is to analyze the best method to identify tweets which talk about tobacco in an anecdotal manner, which can be used to understand the semantic similarity between such tweets. Table 3 illustrates the results for this experiment.

\subsection{Experiment 2: Identifying Tobacco-related Mentions}

The next experiment in the study was to detect all tobacco-related tweets related. These include the following categories of tweets: personal mentions of tobacco-use, general information about tobacco or its use, advertisements. Thus, the experiment was to determine whether the tweet belonged to one of the above categories or not. The objective here is also to gauge semantic information in tweets with mentions of tobacco, suggesting that tweets using the similar slang might be talking about other drugs or ambiguous or unrelated information. Table 3 illustrates the results for this experiment.

\subsection{Experiment 3: Performing Fine-grained Classification of Tobacco-related Mentions}

The last experiment conducted in the study was to classify the tweets into all five categories: UM, PM, IM, AD, OD. Table 4 illustrates the results of the experiment. This is essentially the fine grained classification experiment which relies on semantic information as well as lexical choice. We see that models from all the three experiments perform differently given the type of task. Table 4 illustrates the results for this experiment.

\section{Discussion}

In this section, we analyze our contributions from the perspective of advancing work in the fields of topical content analysis as well as the study of public health mentions in tweets, with regards to tobacco products, as well as e-cigarettes and related products. Given the effects of both as well as the significant overlap in the demographic of consumers of tobacco products and Twitter users, we found it necessary to understand the nature of the tweets produced and consumed by them.

Our dataset, a collection of 3144 tweets, accumulated and filtered over the period of just a week, implies that tobacco and related drugs are tweeted about and spoken of quite frequently, but 


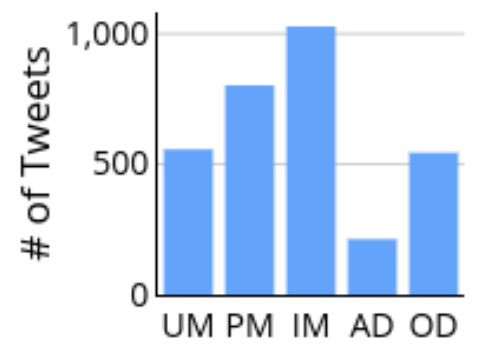

Class

Figure 2: Distribution of tweets among different categories

\begin{tabular}{lll} 
Category & Retweets & Favorites \\
\hline$U M$ & 1079.05 & 0.794 \\
$P M$ & 12171.60 & 0.904 \\
$I M$ & 680.24 & 3.918 \\
$A D$ & 140.81 & 4.586 \\
$O D$ & 873.08 & 0.868
\end{tabular}

Table 5: Average retweets and favorites across classes

the linguistic cues common among these tweets was not considered until now. The inclusion of tweets into the corpus based on slang terminology is an attempt to analyze the Twitter landscape in the language of the audience which most highly correlates with the demographic of consumers for the aforementioned products. To the best of our knowledge, using common slang as a basis of dataset creation and filtration for this task has not been attempted before.

Contemporary methods in the field focus on two basic characterizations, user based and sentiment based. User based classification such as Malik et al. (2019) and Jo et al. (2016) are based on the analyzing activity from a particular user or set of users, while sentiment based analyses such as Paul and Dredze (2011); Allem et al. (2018) and Myslín et al. (2013) are based on understanding the sentiment of the users on the basis of a new product, category or a more generalized perception of smoking in general. On the other hand, public health mention research such as Jawad et al. (2015) focuses on effect of a particular type of tweet, generally health campaigns. Fundamen- tally, the classes we have chosen for the collected data are based on the same principle as the data collection mechanism, with the aim to bridge the gap between the classification studies and the public health surveillance research. This is because our categories cover the breadth of the tweets evenly, directed towards semantically understanding the nature of the tweets. This information is vital for addressing the validity and reach of campaigns, advertisements and other efforts.

Figure 2 shows the distribution of the number of tweets in each class. We see that in the span of a week, informative or advisory and personal mentions are the most widely posted. The tweets that provide general information about smokers or the habits of smoking tobacco or e-cigarettes are generated the most, implying that a larger section of the population tweets of smoking in an anecdotal manner. Similarly, Table 5 shows an interesting trends for the favorites. Advertisements have a higher average favorite count than most other classes, while anecdotal and advisory tweets are the most retweeted on average. This difference is an interesting observation, primarily because on further work such as sentiment analysis and doing short text style transfer (Luo et al., 2019) for these categories may provide an effective strategy for advertisers and campaigners alike.

\section{Conclusion and Future Work}

In this paper, we created a dataset of tweets and classified them in order to understand the social media atmosphere around tobacco, e-cigarettes and other related products. Our schema for categorization targets posts on public health as much as tobacco related products, therefore allowing us to know the number and type of tweets used in public health surveillance for the above mentioned products. Most importantly, we consider slang as a very important aspect of our data collection mechanism, which has allowed us to factor in the content which is circulated and exposed to the majority of the consumers of social media and the aforementioned products both.

This contribution can be further extended by working with other social media platforms, where the methods introduced above can be easily replicated. Social media specific slang can be taken into account to make a more robust dataset for this task. Furthermore, on the public health surveillance aspect, more metadata using the tweets can 
be extracted, which gives an idea of the type of tweets or posts needed to grab the attention of a wider audience on topics of public health and awareness for the grave topic of tobacco products and e-cigarettes.

\section{References}

Jon-Patrick Allem, Likhit Dharmapuri, Adam Leventhal, Jennifer Unger, and Tess Cruz. 2018. Hookahrelated posts to twitter from 2017 to 2018: Thematic analysis. Journal of Medical Internet Research, 20:e11669.

Eiji Aramaki, Sachiko Maskawa, and Mizuki Morita. 2011. Twitter catches the flu: detecting influenza epidemics using twitter. In Proceedings of the conference on empirical methods in natural language processing, pages 1568-1576. Association for Computational Linguistics.

Piotr Bojanowski, Edouard Grave, Armand Joulin, and Tomas Mikolov. 2016. Enriching word vectors with subword information. Transactions of the Association for Computational Linguistics, 5:135-146.

Patricia A. Cavazos-Rehg, Melissa J. Krauss, Shaina Sowles, and Laura J. Bierut. 2015. "hey everyone, i'm drunk." an evaluation of drinking-related twitter chatter. Journal of studies on alcohol and drugs, 76 4:635-43.

Corinna Cortes and Vladimir Vapnik. 1995. Supportvector networks. Mach. Learn., 20(3):273-297.

Daniel K Cortese, Glen Szczypka, Sherry Emery, Shuai Wang, Elizabeth Hair, and Donna Vallone. 2018. Smoking selfies: using instagram to explore young women's smoking behaviors. Social Media+ Society, 4(3):2056305118790762.

Hongying Dai and Jianqiang Hao. 2017. Mining social media data for opinion polarities about electronic cigarettes. Tobacco control, 26(2):175-180.

Xiangfeng Dai, Marwan Bikdash, and Bradley Meyer. 2017. From social media to public health surveillance: Word embedding based clustering method for twitter classification. In SoutheastCon 2017, pages 1-7. IEEE.

Jacob Devlin, Ming-Wei Chang, Kenton Lee, and Kristina Toutanova. 2019. BERT: pre-training of deep bidirectional transformers for language understanding. In Proceedings of the 2019 Conference of the North American Chapter of the Association for Computational Linguistics: Human Language Technologies, NAACL-HLT 2019, Minneapolis, MN, USA, June 2-7, 2019, Volume 1 (Long and Short Papers), pages 4171-4186.
Joseph L. Fleiss and Jacob Cohen. 1973. The equivalence of weighted kappa and the intraclass correlation coefficient as measures of reliability. Educational and Psychological Measurement, 33(3):613619.

Kunihiko Fukushima. 1988. Neocognitron: A hierarchical neural network capable of visual pattern recognition. Neural Networks, 1(2):119-130.

Alex Graves, Abdel rahman Mohamed, and Geoffrey E. Hinton. 2013. Speech recognition with deep recurrent neural networks. 2013 IEEE International Conference on Acoustics, Speech and Signal Processing, pages 6645-6649.

Alex Graves and Jürgen Schmidhuber. 2005. Framewise phoneme classification with bidirectional lstm and other neural network architectures. Neural networks : the official journal of the International Neural Network Society, 18 5-6:602-10.

Shona Hilton, Heide Weishaar, Helen Sweeting, Filippo Trevisan, and Srinivasa Vittal Katikireddi. 2016. E-cigarettes, a safer alternative for teenagers? a uk focus group study of teenagers' views. $B M J$ Open, 6(11).

Sepp Hochreiter and Jürgen Schmidhuber. 1997. Long short-term memory. Neural Computation, 9:17351780.

Mohammed Jawad, Jooman Abass, Ahmad Hariri, and Elie A Akl. 2015. Social media use for public health campaigning in a low resource setting: the case of waterpipe tobacco smoking. BioMed research international, 2015.

Keyuan Jiang, Shichao Feng, Qunhao Song, Ricardo A Calix, Matrika Gupta, and Gordon R Bernard. 2018. Identifying tweets of personal health experience through word embedding and lstm neural network. BMC bioinformatics, 19(8):210.

Catherine L Jo, Rachel Kornfield, Yoonsang Kim, Sherry Emery, and Kurt M Ribisl. 2016. Pricerelated promotions for tobacco products on twitter. Tobacco control, 25(4):476-479.

Armand Joulin, Edouard Grave, Piotr Bojanowski, and Tomas Mikolov. 2016. Bag of tricks for efficient text classification. In EACL.

Diederik P. Kingma and Jimmy Ba. 2014. Adam: A method for stochastic optimization. Cite arxiv:1412.6980Comment: Published as a conference paper at the 3rd International Conference for Learning Representations, San Diego, 2015.

Yann Lecun, Léon Bottou, Yoshua Bengio, and Patrick Haffner. 1998. Gradient-based learning applied to document recognition. In Proceedings of the IEEE, pages $2278-2324$. 
Brianna Lienemann, Jennifer Unger, Tess Cruz, and Kar-Hai Chu. 2017. Methods for coding tobaccorelated twitter data: A systematic review. Journal of Medical Internet Research, 19:e91.

Fuli Luo, Peng Li, Pengcheng Yang, Jie Zhou, Yutong Tan, Baobao Chang, Zhifang Sui, and Xu Sun. 2019. Towards fine-grained text sentiment transfer. In Proceedings of the 57th Conference of the Association for Computational Linguistics, pages 2013-2022.

Aqdas Malik, Yisheng Li, Habib Karbasian, Juho Hamari, and Aditya Johri. 2019. Live, love, juul: User and content analysis of twitter posts about juul. American Journal of Health Behavior, 43:326-336.

Dale S Mantey, Cristina S Barroso, Ben T Kelder, and Steven H Kelder. 2019. Retail access to e-cigarettes and frequency of e-cigarette use in high school students. Tobacco Regulatory Science, 5(3):280-290.

Tomas Mikolov, Anoop Deoras, Daniel Povey, Lukás Burget, and Jan ernocký. 2011. Strategies for training large scale neural network language models. 2011 IEEE Workshop on Automatic Speech Recognition Understanding, pages 196-201.

Tomas Mikolov, Martin Karafiát, Lukás Burget, Jan ernocký, and Sanjeev Khudanpur. 2010. Recurrent neural network based language model. In INTERSPEECH.

Mark Myslín, Shu-Hong Zhu, Wendy Chapman, and Mike Conway. 2013. Using twitter to examine smoking behavior and perceptions of emerging tobacco products. Journal of medical Internet research, 15:e174.

Michael J. Paul and Mark Dredze. 2011. You are what you tweet: Analyzing twitter for public health. In ICWSM. The AAAI Press.

Jeffrey Pennington, Richard Socher, and Christopher Manning. 2014. Glove: Global vectors for word representation. In Proceedings of the 2014 conference on empirical methods in natural language processing (EMNLP), pages 1532-1543.

Tevah Platt, Jodyn Platt, Daniel Thiel, and Sharon L. R Kardia. 2016. Facebook advertising across an engagement spectrum: A case example for public health communication. JMIR Public Health and Surveillance, 2:e27.

Judith Prochaska, Cornelia Pechmann, Romina Kim, and James Leonhardt. 2012. Twitter $=$ quitter? an analysis of twitter quit smoking social networks. Tobacco Control, 21:447-449.

Gerard Salton and Christopher Buckley. 1988. Termweighting approaches in automatic text retrieval. Inf. Process. Manage., 24(5):513-523.

Danielle Sharpe, Richard S Hopkins, Robert Cook, and Catherine Striley. 2016. Evaluating google, twitter, and wikipedia as tools for influenza surveillance using bayesian change point analysis: A comparative analysis. JMIR Public Health and Surveillance, 2:e161.

Jennifer Unger, Daniel Soto, and Adam Leventhal. 2016. E-cigarette use and subsequent cigarette and marijuana use among hispanic young adults. Drug and Alcohol Dependence, 163.

Elizabeth A Vandewater, Stephanie L Clendennen, Emily T Hébert, Galya Bigman, Christian D Jackson, Anna V Wilkinson, and Cheryl L Perry. 2018. Whose post is it? predicting e-cigarette brand from social media posts. Tobacco regulatory science, 4(2):30-43.

Ashish Vaswani, Noam Shazeer, Niki Parmar, Jakob Uszkoreit, Llion Jones, Aidan N Gomez, Łukasz Kaiser, and Illia Polosukhin. 2017. Attention is all you need. In Advances in Neural Information Processing Systems, pages 5998-6008. 